\title{
Peak-hour metro rail traffic congestion alleviation
}

\author{
S. Narayanaswami \\ Indian Institute of Management, Ahmedabad, India
}

\begin{abstract}
An urban light rail transport in the Middle East which is operated as a cyclic schedule on fixed Origin-Destination (O-D) pairs and two lines is considered. A high ridership is observed during weekends and peak hours, leading to severe over-crowding. To reduce over-crowding and alleviate congestions, three different strategic solutions are considered. (i) Reduce peak hour headways. (ii) Increase number of cabins in trains operated during peak hours. (iii) Increase number of services between modified O-D pairs with denser traffic. The problem domain is modelled as a directed transit network and further as an Linear-programming (LP) formulation with a focus on the third solution for one of the lines, under an assumption of known demand. Nodes represent arrival/departure times at each station and constraints on (i) consistency of flow, (ii) non-negativity of flow, (iii) indivisibility, (iv) demand satisfiability and (v) bounds are proposed. The objective is: (i) To minimize number of train cabins in the system; and (ii) to minimize the total car miles run by all trains in a schedule cycle. The LP Model developed for the third proposed solution is tested with actual data using an open LP Solver. Several interesting questions have arisen out of the proposed model. Attempts continue (i) to acquire, test and evaluate validated data from the operators, (ii) simulate the proposed model to one of the two operational lines and study congestion alleviation of both lines in a combined manner and (iii) evaluate the model against other possible solutions.

Keywords: congestion alleviation, cyclic timetabling, LP modeling, peak-hour traffic.
\end{abstract}




\section{Introduction}

A congestion situation arising in a Metro service operated as Light Rail Transport (LRT) with 5 cabin trains in the Middle East is considered in this model. Trains are automatically operated with the Red line running between RRT (Red Right Terminal) and RLT (Red Left Terminal) and (ii) the Green line operating between GRT (Green Right Terminal) and GLT. Two yards at RRT (Red Right Terminal) and RLT (Red Left Terminal) serve the red line trains and one yard at GRT (Green Right Terminal) serves the green line trains. Alignment plans and train operational schedules are known. Operational times are based on week days and weekends and the headways are based on peak hour and off-peak hours of the day. Light rails are used in this metro and each train has five cabins. On both lines, headways are designed at 90 seconds, whereas currently operated headway during peak hours is 3.5 minutes. Operational hours are based on days of the week and headways between the trains are based on hours of the day. The entire system is designed to handle a 90 seconds headway, though the peak hour traffic is operated at 3.5 minutes. It is observed that the peak hour demands are very high in both lines and particularly during the week ends. To alleviate congestion, three types of solutions are considered. (i) Reduce peak hour headways (ii) Increase number of cabins in trains operated during peak hours. (iii) Increase number of services between modified O-D pairs with denser traffic. The two lines operate between fixed O-D pairs and peak hour traffic is generally directed at one direction in the morning and the opposite in the evening. For that reason, reducing peak hour headway shall reduce the number of empty runs in the reverse direction and hence is not a favored solution. Increasing the number of cabins in a train shall not be allowed, as some stations are limited in the number of cabins that can be accommodated. G1 (RG1) and RG2 are the two cross-over stations between the two lines. Based on ridership data available over the years, traffic is highest between R1 and R2 on the red line and between G1 (RG1) and G2 on the green line. Interestingly peak hour traffic is dense around two bottle neck stations, R1 and R2 on the red line and around G1 (RG1) and G2 on the green line. The third option is being considered to offer additional services in the bottle neck corridor between additional O-D pairs. At the present level of implementation, only the red line is being tested and evaluated.

Economists perceive congestion management [1] in terms of differential pricing, whereas operationally a surge in demand is a lost revenue and excess capacity is revenue wastage. A review of congestion management models specific to light rails is presented by [2]. Particularly a model that is built on mathematical approach is presented in [3] and the one based on simulation approach is presented by [4].

\section{Problem statement}

Peak-hour traffic between certain stations on the red line and green line lead to enormous congestion and passenger discomfort. To alleviate congestion and ease traffic mobility, it is proposed to consider an additional O-D pair on the red line between R1 and R2 and to operate trains between these corridors with smaller 
headways during peak hour traffic. The problem is to find out the minimum number of trains required to transport the passengers between the bottle neck stations during peak hours. The objectives proposed to the problem are namely (i) to minimize the number of train cabins in the system and (ii) to minimize the total car miles run by all trains in a schedule cycle under the constraints of (i) consistency of flow, (ii) non-negativity of flow, (iii) indivisibility, (iv) demand satisfiability and (v) bounds. It is further assumed that demand during peak hours is known. An LP Model is developed for the third proposed solution and evaluated with actual data extracted from the read line in September 2012 using an open package LP solver package for Win32, namely GUSEC (GLPK Under Scite Extended Kit).

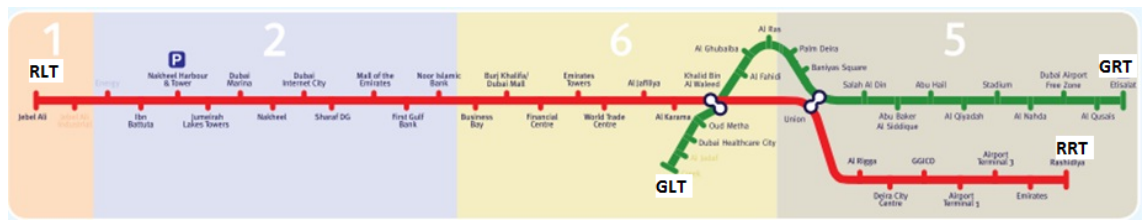

Figure 1: The Redline route between RRT and RLT.

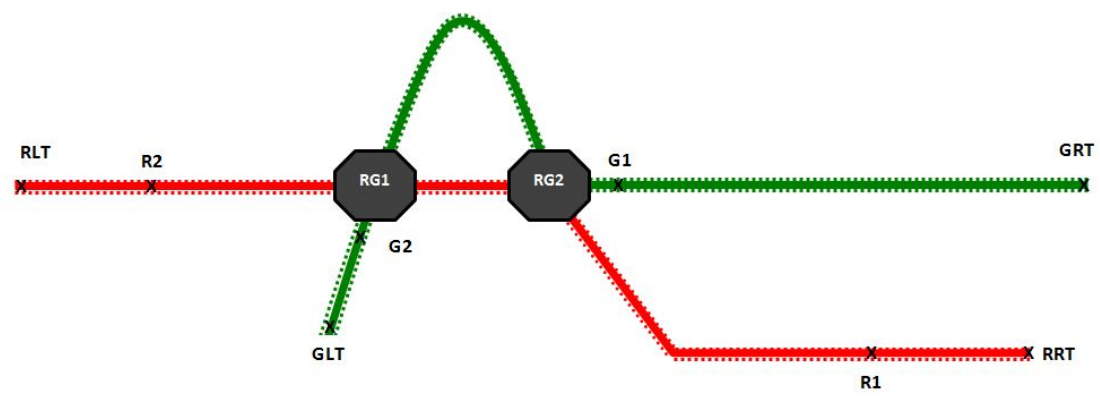

Figure 2: The modified O-D pairs of R1 and R2 station.

\section{Formulation of the model}

The proposed model is motivated by the railroad network and in this section, the network that applies to the problem statement of the metro operations is completely described. A fleet of cabins provides transport service between two fixed O-D pairs on the red and green lines. A set of trains is scheduled on both lines, each comprising 5 cabins each. At any given time, cabins are either part of running trains or stored at one of three yards. Two requirements restrict the size and deployment 
of cabins, and hence the trains: one is the fixed schedule and second is the known demands for scheduled trains.

Fixed Schedule: It is a list of all trains that depart in a schedule cycle. An entry in the list specifies each city of departure and arrival and the corresponding departure and arrival times. In the congestion alleviation problem of the metro operator, we consider the cyclic schedule of red line on Thursdays.

Demands: For each scheduled train, there is a known demand which must be met. A minimum number of cabins is required in each train.

In the following two tables, ridership in the red line in either directions (RRT to RLT and RLT to RRT) are given.

Table 1: Capacity during weekdays.

\begin{tabular}{||llc||}
\hline \multicolumn{3}{|c||}{ Weekday: RRT to RLT } \\
\hline Schedule period & Headways & Number of cabins \\
\hline 0550 to 0630 & 7 Minutes & 21 \\
0630 to 0900 & 3.5 Minutes & 41 \\
0900 to 1530 & 8 Minutes & 18 \\
1530 to 1740 & 7 Minutes & 21 \\
1740 to 1900 & 3.5 Minutes & 41 \\
1900 to 2000 & 7 Minutes & 21 \\
2000 to 2300 & 8 Minutes & 18 \\
2300 to 0000 & 10 Minutes & 14 \\
\hline & Weekday: RLT to RRT \\
\hline Schedule period & Headways & Number of cabins \\
\hline 0550 to 0900 & 7 Minutes & 21 \\
0900 to 1515 & 8 Minutes & 18 \\
1515 to 1630 & 7 Minutes & 21 \\
1630 to 1900 & 3.5 Minutes & 41 \\
1900 to 2200 & 8 Minutes & 18 \\
2200 to 0000 & 10 Minutes & 14 \\
\hline
\end{tabular}


Table 2: Capacity on the last day of the week.

\begin{tabular}{||lll||}
\hline \multicolumn{3}{|c||}{ Thursday: RRT to RLT } \\
\hline Schedule period & Headways & Number of cabins \\
\hline 0550 to 0630 & 7 Minutes & 21 \\
0630 to 0900 & 3.5 Minutes & 41 \\
0900 to 1530 & 8 Minutes & 18 \\
1530 to 1740 & 7 Minutes & 21 \\
1740 to 1900 & 3.5 Minutes & 41 \\
1900 to 2200 & 8 Minutes & 18 \\
2000 to 2300 & 8 Minutes & 18 \\
2300 to 0000 & 10 Minutes & 14 \\
\hline & Thursday: RLT to RRT & \\
\hline Schedule period & Headways & Number of cabins \\
\hline 0550 to 0630 & 7 Minutes & 21 \\
0630 to 0900 & 3.5 Minutes & 41 \\
0900 to 1515 & 8 Minutes & 18 \\
1515 to 1630 & 7 Minutes & 21 \\
1630 to 2000 & 3.5 Minutes & 41 \\
2000 to 2300 & 8 Minutes & 18 \\
2300 to 0000 & 10 Minutes & 14 \\
\hline
\end{tabular}

\subsection{Ridership details}

- September - Red Line

Weekday PPHPD AM Peak - 5408

Weekday PPHPD PM Peak - 6620

Weekday PPHPD off peak - 1800 approx

Thursday PPHPD AM Peak - 5673

Thursday PPHPD PM Peak - 7067

Thursday PPHPD off peak - 2000 approx

Friday PPHPD - 2500 approx

Saturday PPHD - 3500 approx 
Table 3: Capacity during weekends.

\begin{tabular}{||lcc||}
\hline \multicolumn{3}{||c||}{ Friday: Operational hours on both directions } \\
\hline Schedule period & Headways & Number of cabins \\
\hline 1300 to 0100 & 8 Minutes & 18 \\
\hline Saturday: Operational hours on both directions \\
\hline Schedule period & Headways & Number of cabins \\
\hline 0550 to 0100 & 8 Minutes & 18 \\
\hline
\end{tabular}

Table 4: Capacity on Green line.

\begin{tabular}{||lcc||}
\hline \multicolumn{3}{|c||}{ Green Line: Week day and Saturday } \\
\hline Schedule period & Headways & Number of cabins \\
\hline 0500 to 0000 & 8 Minutes & 10 \\
\hline \multicolumn{3}{|c||}{ Green Line: Thursday } \\
\hline 0550 to 0100 & 8 Minutes & 10 \\
\hline \multicolumn{3}{|c||}{ Green Line: Friday } \\
\hline 1300 to 0100 & 8 Minutes & 10 \\
\hline
\end{tabular}

- Green Line

$$
\begin{aligned}
& \text { Weekday - } 1600 \text { max } \\
& \text { Thursday - } 1600 \text { max } \\
& \text { Friday - } 1400 \text { max } \\
& \text { Saturday - } 1400 \text { max }
\end{aligned}
$$

In the above list, PPHPD denotes the unit of ridership in Passengers Per Hour Per Direction (PPHPD). A total of 79 trains, each with 5 cabins are deployed. A total of 16 trains spares in red line (80 cabin spares on red line) and 5 trains spares on green line (25 cabin spares) are available. We consider the stations, R1 and R2 stations on the redline and the number of passengers that travelled between the stations in September, 2012. The running capacities of all services during week days and week ends are tabulated in Tables 1, 2, 3 and 4. 


\subsection{Transit network model}

We define one node for each city in the network for each time. The schedule period is divided into $\tau$ uniform intervals. We have considered $\tau=144$, which means $T=\{0,1,2, \ldots 144\}$ and each time interval at every ten minutes is considered. We connect the nodes by arcs of two types, by cabins in storage and cabins in trains. We assume the following in our proposed model.

- Demand at each city (station) and at each interval is known.

- Dedicated tracks are assumed in each direction of train movement.

- No train is allowed to overtake any other train.

- Headway is maintained.

Storage arcs: For each city, run an arc from the node for each time $t$ to the node for the next time, $(t+1) \bmod \tau$. This would give the number of cabins available as storage cabins in the city during that interval at the beginning of $t$.

Train arcs: For each scheduled train, run an arc from the node representing the city and time of departure to the node for the city and time of arrival. Flow along this arc represents cabins moving from one city to another in the scheduled train.

\subsection{Constraints}

Consistency of flow: This constraint ensures that the net flow at every node is zero. The number of cabins in storage during interval $t$ at a given city must equal the number in storage in the interval immediately before, plus the number that arrived at time $t$ less the number that departed at $t$.

Non-negativity of flow: All flows are non-negative. This ensures that trains cannot move backwards in time and space.

Indivisibility: Cabins are in indivisible units, and hence all flows must be integral.

Demand satisfiability: The flow on each train arc must be greater than or equal to the number of cabins required to meet demands.

Bounds: This constraint limits the maximum and minimum number of cabins in a train. The operator does not allow flexibility in the number of cabins attached to a train. It is a fixed 5-cabin train, as of now. But as a generic model, it is possible to vary the number of cabins.

\subsection{Reducing the network}

If no trains arrive or depart city $c$ at time $t$, the city is connected to the rest of the network by only two storage arcs: an incoming arc from the previous time and outgoing arc to the following time. These flows must be equal to satisfy the consistency of flow constraint. Consequently this arc may be removed and the two arcs may be replaced with one. The redline network is reduced based on the above discussions and the modified O-D pairs are considered. At the present level of 
implementation, green line is not considered in our computation, though we have illustrated in the figure.

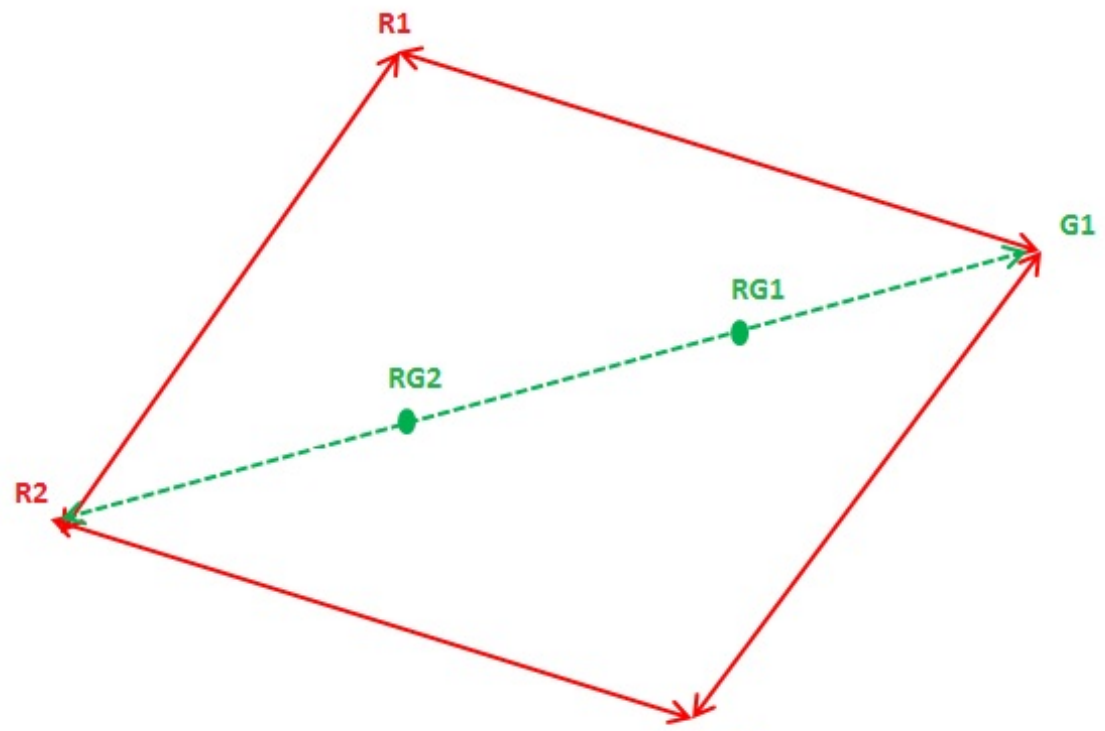

G2

Figure 3: Reduced network model of RRT-RLT on redline.

\subsection{LP formulation}

Notation: The notation of sets and variables used in the proposed model is listed below:

- $C$ : The set of cities; $C=\{A, B, C, D\}$

- $T$ : Set of intervals into which the schedule period is divided; $T=$ $\{0,1,2,3, \ldots \tau\}$

- $S$ : The known schedule; $S=\left\{\left(c, t, c^{\prime}, t^{\prime}\right): c \in C, c^{\prime} \in C, t \in T, t^{\prime} \in\right.$ $T$ and $\left.c \neq c^{\prime}\right\}$

An element in $S$ represents a train that leaves city $c$ at time $t$ and arrives at $c^{\prime}$ at $t^{\prime}$.

- $d_{c c^{\prime}}{ }^{t t^{\prime}}$ : Smallest number of cabins required to meet demand for train $\left(c, t, c^{\prime}, t^{\prime}\right)$

$$
d_{c c^{\prime}} t t^{\prime} \geq 0
$$

- $h_{c c^{\prime}} t t^{\prime}$ : Maximum number of cabins required to meet demand for train $\left(c, t, c^{\prime}, t^{\prime}\right)$

$$
h_{c c^{\prime}} t t^{\prime} \geq 0 \text {. }
$$


- $N_{c}(t)$ : Nodes of the network, $\forall c \in C$ and $t \in T$

- $A S_{c}{ }^{t}$ : Directed arcs that represent storage of unused cabins, $\forall c \in C$ and $t \in T$

$$
A S_{c}{ }^{t}: N_{c}(t) \rightarrow N_{c}((t+1) \bmod \tau)
$$

$a s_{c}{ }^{t}:$ LP variable that defines train flow over $A S_{c}{ }^{t}, \forall c \in C$ and $t \in T$

- $A M_{c c^{\prime}}{ }^{t t^{\prime}}$ : Directed arcs that represent movement of cabins, $\forall c, c^{\prime} \in$ $C$ and $t, t^{\prime} \in T$

$$
A M_{c c^{\prime}} t t^{\prime}: N_{c}(t) \rightarrow N_{c^{\prime}}\left(t^{\prime}\right)
$$

$a m_{c c^{\prime}} t t^{\prime}:$ LP variable that defines train flow over $A M_{c c^{\prime}} t t^{\prime}, \forall$ $c, c^{\prime} \in C$ and $t, t^{\prime} \in T$

- $m_{c c^{\prime}}$ : Distance in miles from $c$ to $c^{\prime}$

Constraints: The list of constraints in LP notation is given below.

1. Consistency of flow:

$$
a s_{c}^{t-1 \mid \tau}+\sum_{(c 1, t 1, c, t) \in S} a m_{c 1 c}{ }^{t 1 t}=a s_{c}{ }^{t}+\sum_{(c, t, c 2, t 2) \in S} a m_{c c 2}{ }^{t 2}
$$

$\forall c \in C$ and $t \in T$

For every city and time, unused cabins in the present interval must equal unused cabins in the previous interval, plus cabins just arriving in trains, minus cabins just leaving in trains.

2. Non-negativity of flow:

$$
a s_{c}{ }^{t} \geq 0
$$

$\forall c \in C$ and $t \in T$

Cabins are not permitted to run in the reverse direction.

3. Indivisibility:

$$
\begin{gathered}
a s_{c}{ }^{t} \in\{0,1\} \\
a m_{c c^{\prime}} t t^{\prime} \in\{0,1\}
\end{gathered}
$$

$\forall c \in C$ and $t \in T$

Cabins are indivisible.

4. Demand satisfiability:

$$
a m_{c c^{\prime}} t t^{\prime} \geq d_{c c^{\prime}} t t^{\prime}
$$

$\forall\left(c, t, c^{\prime}, t^{\prime}\right) \in S$

For each scheduled train, number of cabins must meet demand, but must not be so great that unnecessary cabins are run. 
5. Bounds:

$$
\begin{gathered}
h_{c c^{\prime}}^{t t^{\prime}} \geq d_{c c^{\prime}}^{t t^{\prime}} \\
d_{c c^{\prime}}^{t t^{\prime}} \leq a m_{c c^{\prime}}^{t t^{\prime}} \leq h_{c c^{\prime}}^{t t^{\prime}}
\end{gathered}
$$

Minimum and maximum number of cabins are specified for a given demand between cities at a given time interval.

Objective Function: Based on the problem statement and the set of constraints, a feasible set of cabin allocations may be determined for each train in the schedule, if one exists. Here we propose a linear objective function to optimize such an allocation of the train flow variables. A case of two objectives is developed, for a schedule period of one day. A set of solution activities of $a s_{c}{ }^{t}$ and $a m_{c c^{\prime}} t t^{\prime}$ variables is written as (as, am) and the value of the objective function $Z$ at the solution is $Z$ (as, am). Two costs are proposed and the objective is to minimize the total cost. The first cost is fleet cost, directed at minimizing the number of cabins in the fleet. The second cost is the operating cost, directed at minimizing the number of cabin-miles. Let at $t^{*}, 0 \leq t^{*} \leq(\tau-1)$, the total number of cabins in the system be determined. For a feasible solution, this number must be same at any $t^{*}$, since cabins do not enter or leave an operational system. Let us consider, $t^{*}=\tau-1$. Then the total number of cabins is given by,

$$
Z_{\text {fleet }}=\sum_{\left(c, t, c^{\prime}, t^{\prime}\right) \in S} a m_{c c^{\prime}} t t^{\prime}+\sum_{c \in C} a s_{c}{ }^{\tau-1}
$$

Let $m_{c c^{\prime}}$ be the distance from $c$ to $c^{\prime}$. Then total cabin miles in given by,

$$
Z_{\text {mile }}=\sum_{c, t, c^{\prime}, t^{\prime} \in S} m_{c c^{\prime}} X a m_{c c^{\prime}} t t^{\prime}
$$

The objective function is to minimize total cost $Z$ and is given by

$$
\text { Minimize } Z(\mathbf{a s}, \mathbf{a m})=p_{1} X Z_{\text {fleet }}+p_{2} X Z_{\text {mile }}
$$

where $p_{1}$ (Cost / Fleet) and $p_{2}$ (Cost / Fleet-Mile) are the constants of proportionality of the costs $Z_{\text {fleet }}$ and $Z_{\text {mile }}$ respectively.

\section{Implementation}

The Metro transport service operates with fixed O-D pairs, which is RRT and RLT. To maintain consistency of evaluation, results are compared between the two bottleneck stations, R1 and R2 stations. The model is implemented and three different variations are reported. (i) Data 1 is the currently operated schedule and demands. (ii) Data 2 is a proposed capacity increase of number of trains run between the given O-D pair, by manually increasing the number of cabins in movements during the peak hour time intervals. (iii) Data 3 is a feasible solution of the model, to determine the number of cabins in movement and storage. Optimality has to verified with more rigorous tests. 


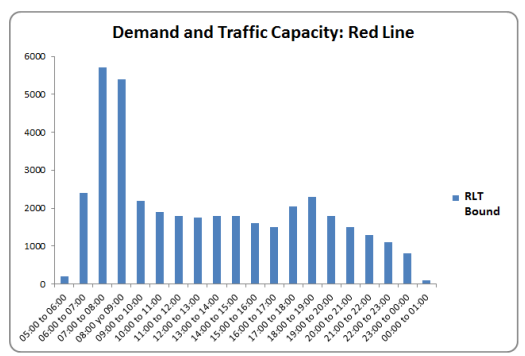

(a) Demand on RRT.

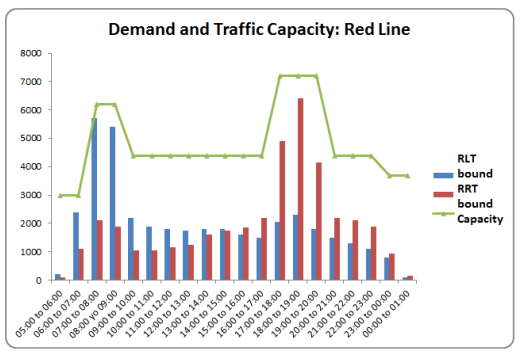

(c) Available capacity.

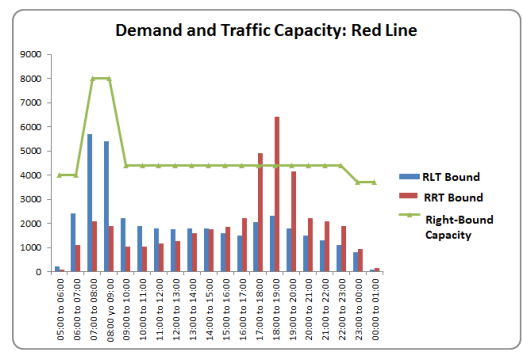

(e) Increasing capacity towards RRT.

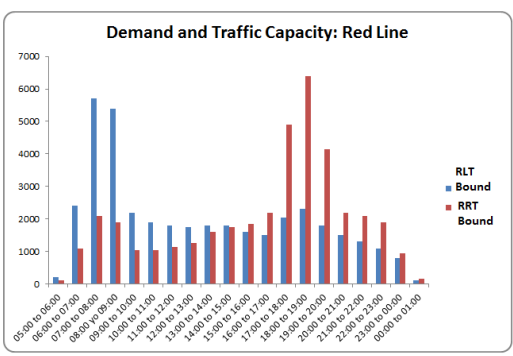

(b) Demand on LRT and RRT.

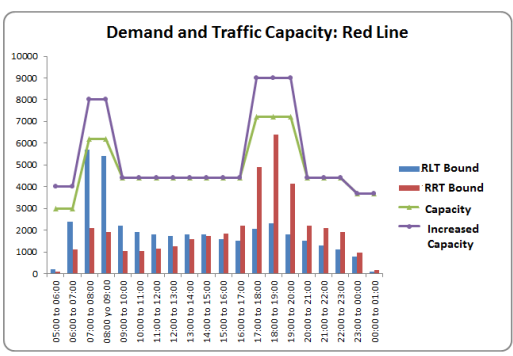

(d) Increased capacity.

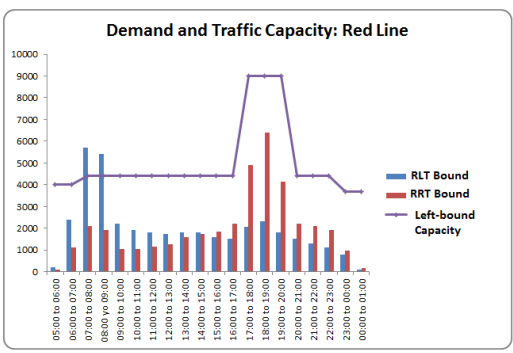

(f) Increasing capacity towards RLT.

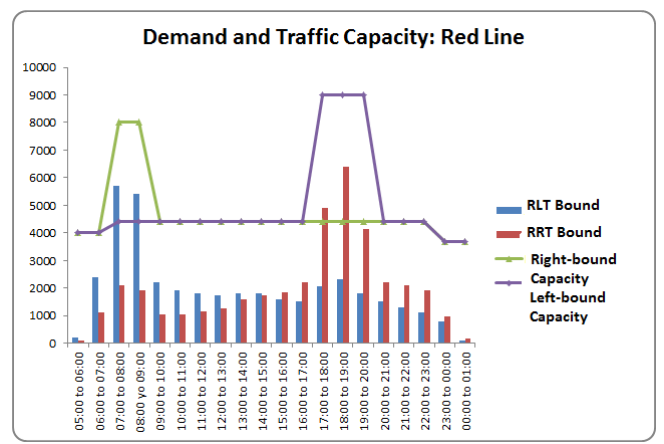

(g) Capacity increase in the proposed model.

Figure 4: Demand fluctuations at various hours of the day. 


\subsection{Computations}

The GUSEC solver was used to solve the LP congestion alleviation model. With the available data of the metro operator ridership, the model was implemented between the densest corridor on the redline, which is R1 - R2 station. Demands on the two lines are plotted in Figures 4(a) and 4(b). Three instances of experimentation was done with the following variations: (i) the currently operated capacity on the redline; it is observed that line is congested during peak hours. Currently available capacity and an overall capacity increase are illustrated on Figures 4(c) and 4(d). (ii) The second set of data proposes an increased capacity during peak hours by reducing the headways during peak hours. Figures 4(e) and 4(f) show the increased capacity towards RLT and RRT during the respective peak traffic. (iii) The third results in the least objective and is illustrated in Figure 4(g), which proposes increasing the capacity (number of services) towards RLT in the morning peak hours and increasing service capacity towards RRT in the evening peak hours.

Table 5: Numerical results of Solving the Congestion Alleviation Model.

\begin{tabular}{||lllrrr||}
\hline Data Sets & Fleet & Miles & $p_{1}$ & $p_{2}$ & Z \\
\hline 1 & 79 & 55000 & 5 & 8 & 440395 \\
\hline 2 & 85 & 89375 & 5 & 8 & 715425 \\
\hline 3 & 75 & 53075 & 5 & 8 & 424975 \\
\hline
\end{tabular}

\section{Summary}

A methodological approach to evaluate congestion alleviation strategies is presented in this paper. Tentative values are used for the two proportionality constants $p_{1}$ and $p_{2}$, which needs to be verified. As can be observed, solutions are highly sensitive to the two proportionality constants. Attempts are on to obtain valid test data from the operator and to evaluate results against other comparable results. Further studies are directed towards extending the model to the green line and determining the combined effect of congestion alleviation in both lines together.

\section{References}

[1] H. Lapan G. Pautsch and C.P. Baumel. An Economic Investigation into Railroad Pricing and Car Allocation Programs. Technical report, Iowa State University, 1995. 
[2] D. Bayraktar S.D. Oncul, D. Aykac and D. Celebin. A Review of Timetabling and Resource Allocation Models For Light-Rail Transportation Systems. Technical report, 2008.

[3] K. Kim and S. Suh. Allocation of Rail Line Capacity between KTX and Conventional Trains under Different Policy Goals with Mathematical Programming. Journal of the Eastern Asia Society for Transportation Studies, 2003.

[4] N. Adamko and V. Klima. Optimisation of Railway Terminal Design and Operations Using Villon Generic Simulation Model. Transport, 23(4):335340, 2008. 